\title{
Opposing needling for analgesia and rehabilitation after unilateral total knee arthroplasty: a randomized, sham- controlled trial protocol
}

\author{
Hai Huang ${ }^{1}$, Xiuling Song ${ }^{2}$, Ling Zhao ${ }^{2}$, Lin Zheng ${ }^{1}$, Lianbo Xiao ${ }^{1 *}$ and Yuelai Chen²
}

\begin{abstract}
Background: This randomized controlled clinical trial aims to evaluate the efficacy and safety of opposing needling in patients undergoing unilateral total knee arthroplasty (TKA). Opposing needling is one of the special needling methods used in acupuncture and moxibustion therapy. It involves needling acupoints on the contralateral side for pain management. Although, opposing needling is used for pain management in clinics, evidence to support its effectiveness as an analgesic after total knee arthroplasty is scant. We designed a randomized controlled clinical trial to evaluate efficacy and safety of opposing electroacupuncture (EA) in alleviating pain associated with unilateral total knee arthroplasty.

Methods/design: This is a protocol for a randomized controlled patient- and assessor-blinded trial with three parallel arms (A, opposing EA; B, operated side EA; C, sham EA). Yinlingquan (SP9), Yanglingquan (GB34), Futu (ST32), and Zusanli (ST36) acupoints are selected for all three groups. In group A, the healthy side will be treated with EA, while the operated side will be administered sham EA. In group B, the operated side will be treated with EA while on the healthy side sham EA will be used. For group C, sham EA will be used on both sides. All patients in the three groups will receive treatment once a day for 3 days. The post-operative pain measured using a visual analogue scale score (including pain while resting and being active) and the additional dose of the patientcontrolled analgesic pump after operation will be recorded as the primary outcomes. Secondary outcomes such as knee function and swelling, range of motion (including active and passive range of motion), post-operative anxiety, and acupuncture tolerance will also be assessed.
\end{abstract}

Discussion: Opposing needling is a potential non-pharmacological treatment for relieving pain and improving functional rehabilitation after TKA, during which patients receive acupuncture on the healthy side rather than on the operated side. This sham controlled clinical trial, designed to evaluate efficacy and safety of opposing needling for patients after TKA, will provide evidence for pain management and functional rehabilitation after unilateral TKA.

\footnotetext{
*Correspondence: xiao_lianbo@163.com; chen_yuelai@163.com

'Guanghua Hospital, Shanghai University of Traditional Chinese Medicine,

540 Xinhua Rd., Shanghai 200052, China

${ }^{2}$ Shanghai University of Traditional Chinese Medicine, 1200 Cailun Rd.,

Shanghai 201203, China
}

(c) The Author(s). 2020 Open Access This article is licensed under a Creative Commons Attribution 4.0 International License, which permits use, sharing, adaptation, distribution and reproduction in any medium or format, as long as you give appropriate credit to the original author(s) and the source, provide a link to the Creative Commons licence, and indicate if changes were made. The images or other third party material in this article are included in the article's Creative Commons licence, unless indicated otherwise in a credit line to the material. If material is not included in the article's Creative Commons licence and your intended use is not permitted by statutory regulation or exceeds the permitted use, you will need to obtain permission directly from the copyright holder. To view a copy of this licence, visit http://creativecommons.org/licenses/by/4.0/. The Creative Commons Public Domain Dedication waiver (http://creativecommons.org/publicdomain/zero/1.0/) applies to the data made available in this article, unless otherwise stated in a credit line to the data. 
(Continued from previous page)

Trial registration: ChiCTR, ChiCTR1800020297 (http://www.chictr.org.cn/edit.aspx?pid=34231\&htm=4). Registered 22 December 2018.

Keywords: Opposing needling, Total knee arthroplasty, Sham acupuncture, Pain, Protocol,

\section{Background}

Total knee arthroplasty (TKA) is one of the best treatments for advanced knee osteoarthritis (KOA) [1], providing a standardized treatment for relieving pain, remodeling knee function, improving quality of life, and improving postural balance [2,3]. Although a number of benefits are associated with TKA, it is imperative to address the potentially increased rate of complications of post-operative pain, post-operative infection, and deep vein thrombosis among these older patients [4]. The management of post-operative pain is a recurring problem that needs effective rectification. Post-operative pain still accounts for $14.1 \%$ of the most common reasons for re-admission within 90 days of surgery as reported in a European study [5]. Thus, post-operative pain is still a major complication that arises after TKA [6]. A number of patients are dissatisfied with the efficacy of commonly used analgesics post-operation and the ancillary side effects that come with them [7, 8]. Kim et al. have focused on the risk factors associated with persistent chronic opioid usage after surgery and suggest that targeted opioid reduction programs may be appropriately implemented to manage this high-risk population [9]. Effective and adequate pain management can restore joint function, prevent chronic pain [10], and allow for a more comfortable and rapid rehabilitation [11].

Acupuncture from ancient Chinese medicine is a safe treatment method without serious side effects [12]. It can be used as a complementary and alternative therapy for many diseases such as dyspepsia [13], overactive bladder [14], stress urinary incontinence [15], etc. Clinically, it can also be used as a drug-free intervention to relieve post-operative pain and help reduce the use of opioid analgesics [16]. Post-operative pain control following TKA using acupuncture therapies has more potential to develop into effective treatment regimens than other alternative therapies [17]. Tedesco et al. [16] systematically evaluates and meta-analyses the drug-free interventions available as post-operative analgesia in the context of TKA. Medium-quality evidence shows that electrotherapy and acupuncture after TKA can reduce the use of opioids and delay opioid consumption. Electroacupuncture (EA) can stimulate acupoints to alleviate pain by linking up a painless weak electrical stimulation. EA not only relieves acute pain [18] but is also effective against chronic pain [19]. This may, therefore, be considered as an adjunct intervention for long-term pain relief in standard non-drug therapy. In Mikashima et al.'s study [20], acupuncture on the affected side provided an effective treatment for post-operative acute pain. And in our previous trial [21] on applying EA for post-operative pain management after TKA, the technique of sham EA was mastered. During our study, however, the researchers were concerned that acupuncture near the incision may increase the risk of post-operative infection, so we considered the treatment of opposing needling after operation.

Opposing needling, also known as healthy side acupuncture or contralateral acupuncture, is one of the nine needling methods of acupuncture first recorded in Huangdi Neijing. According to the patient's condition, the needling method is used at select acupoints on the right side of the body when the left side is affected, and vice versa $[22,23]$. Opposing needling therapy is used in various types of neuralgia and functional rehabilitation therapies in China [24, 25], wherein acupoints are selected generally on the healthy side corresponding to the affected side [26]. Studies [27, 28] have indicated distinct anti-nociceptive effects and mechanisms between ipsilateral and contralateral acupuncture and moxibustion. And anterior cingulate cortex plays an important role in contralateral acupuncture and moxibustion. Alleviation of pain is one of the major areas for the use of opposing needling; however, it has not been used for the treatment of post-operative pain.

Thus, the study protocol that we designed is a clinical study on the opposing needling method for analgesia and rehabilitation after unilateral total knee arthroplasty.

\section{Methods/design \\ Objective}

The aim of this study is to investigate the effectiveness of opposing EA compared with operated side EA or sham EA treatment in patients after unilateral TKA, and to provide clinical evidence for the treatment of postoperative pain by opposing needling.

\section{Study design}

A randomized, controlled, three-arm, patient- and assessor-blinded trial will be conducted to compare the efficacy of two true EA groups (opposing EA and operated side EA) with a sham EA group (Fig. 1).

The study will be sequentially conducted as follows: 114 patients in Guanghua Hospital, Shanghai University 


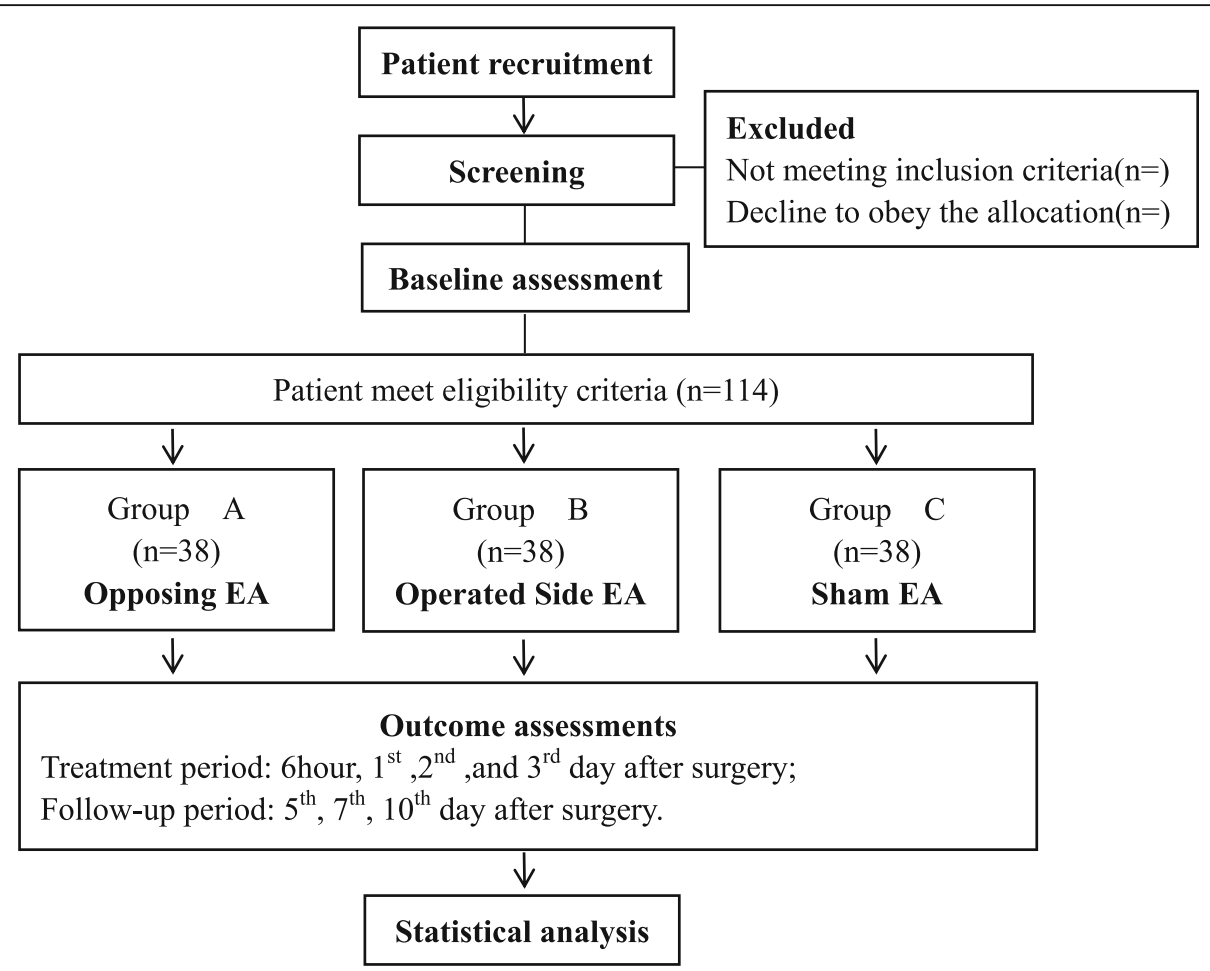

Fig. 1 The flow diagram is intended to depict the passage of participants through this RCT

of Traditional Chinese Medicine with osteoarthritis are included (surgical contraindications are excluded); after admission, unilateral TKA will be performed as scheduled. A day before the surgery, the eligible patients will be assessed at baseline and their consent will be obtained in writing. Patients will then be randomly assigned (on a 1:1:1 ratio) to the opposing EA group, the operated side EA group, or the sham EA group. Random numbers will be generated by the SPSS 22.0 software and sealed in opaque envelopes. Only the operator in charge of acupuncture treatment is authorized to open the envelope to obtain the group code.

The three groups of subjects will receive interventions on three consecutive days after surgery, once every day, by a clinician with more than 3 years of experience in acupuncture intervention. The participants will be blinded to the intervention measures. The success of the visit would be evaluated during the last visit of each participant. Researchers who did not participate in the treatment and who did not know the allocation results will perform the outcome assessment. Unblinding before the completion of the study will only be allowed in the case of medical emergencies or severe adverse events (SAEs).

All patients will receive the same post-operative analgesic dose with a fentanyl patient-controlled analgesic (PCA) pump (continuous infusion rate $0.25 \mu \mathrm{g} /(\mathrm{kg} \cdot \mathrm{h})$ ). Additional analgesics will be provided as per each patient's requirements ascertained by the visual analogue scale (VAS) score $>6$, and the use of analgesics will be recorded in their case report form (CRF).

\section{Eligibility \\ Inclusion criteria}

Subjects are eligible to participate if they meet the following criteria: (1) aged 40 to 75 years; (2) meet the Clinical Classification Criteria for Osteoarthritis of the Knee as recommended by the American College of Rheumatology; (3) undergoing unilateral TKA under general anesthesia during hospitalization without surgical contraindications; (4) American Society of Anesthesiologists (ASA) grade I or II [29].

\section{Exclusion criteria}

Participants will be excluded if they are experiencing or have a history of the following: (1) acupuncture cannot be performed because of skin lesions at acupoints; (2) lower extremity sensory disturbance or abnormality; (3) severe arrhythmias, heart failure, chronic obstructive pulmonary disease, epilepsy, mental disorders; (4) received acupuncture treatment in the past 1 month.

\section{Dropout criteria}

Any participant will be dropped from the study if she or he (1) cannot tolerate acupuncture after operation or cannot complete the study protocol scheduled; (2) cannot continue 
the acupuncture procedure because of surgical factors; (3) refuses to receive EA or sham EA treatment; (4) shows SAEs; (5) violates the protocol or refuses to follow up.

\section{Treatment protocol}

On the basis of the records in the ancient Traditional Chinese Medicine work Huangdi Neijing, treatment for flaccidity is aimed at the yangming meridian (ST). Acupoints in the stomach channel of foot-Yangming are the most involved ones, featuring a distribution around knees. Futu (ST32) and Zusanli (ST36) points on the stomach meridian of foot-Yangming are selected. Furthermore, according to the meridians and acupoints with opposite locations, combined with the principle of local acupoint selection [30], Yinlingquan (SP9) and Yanglingquan (GB34) are selected as a pair of acupoints located, respectively, at yin meridians and yang meridians with opposite locations; it can regulate yin and yang, qi, and blood with the selection of lesser points but with a better effect [22]. After discussion and consensus among acupuncture experts, the above four acupoints have been chosen for the study (Fig. 2).
Patients will stay in a relaxed and supine position before the onset of treatment. The acupuncturist will untie the elastic bandage that was fixed during the operation, so that the acupoints are exposed. The skin around the acupoints is sterilized with cotton dipped in $75 \%$ alcohol, and a sterile adhesive pad (10 $\mathrm{mm}$ in diameter, $5 \mathrm{~mm}$ thick) is pasted on each of the acupoints (Fig. 3). The sterile adhesive pad is made of a special sponge (Suzhou Medical Appliance Factory, China) with one sticky side which can be pasted on the skin. The role of the sterile rubber pad is to fix the location of acupoints and to ensure subject blinding. The participants in the three groups will receive a 30min treatment on the first, second, and third day after operation by the same acupuncturist, three times per session. It will be difficult for them to ascertain which group they belong to.

\section{Opposing EA group}

The healthy side of the lower limbs will be treated with true EA while on the operated side sham EA will be used.
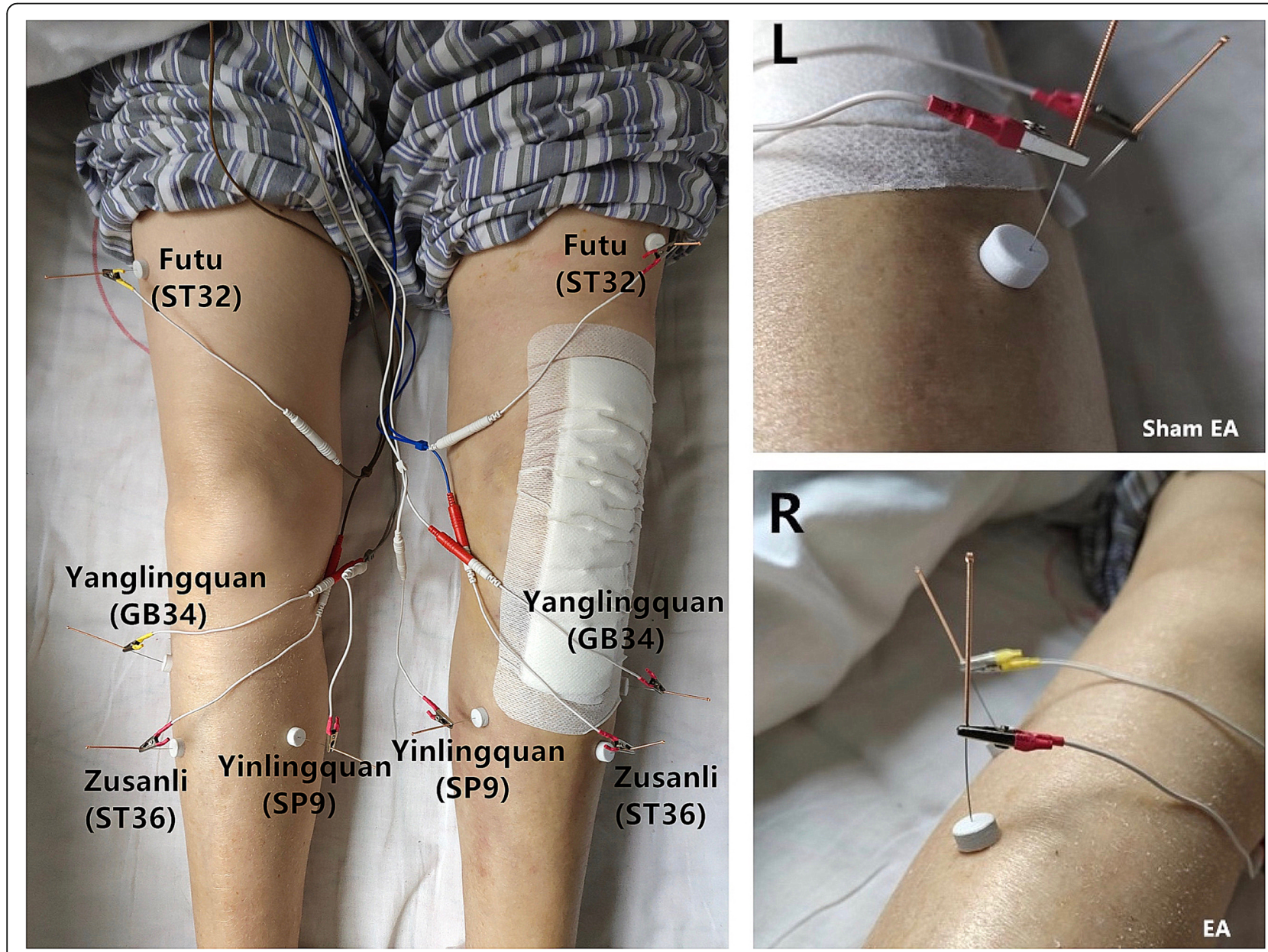

Fig. 2 Acupoints of treatment, EA, and sham EA 


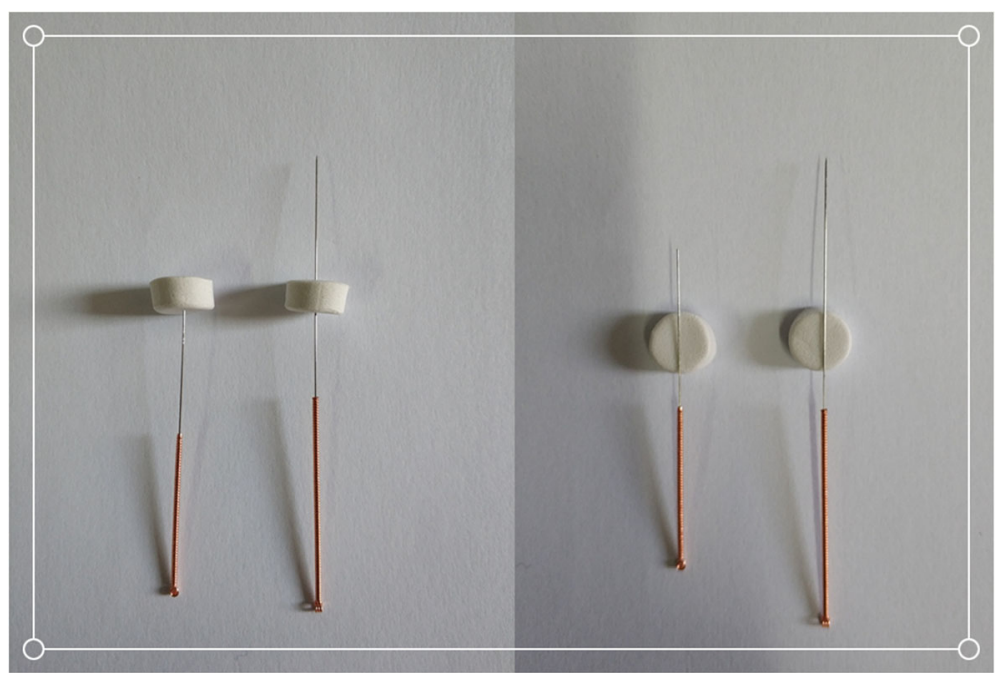

Fig. 3 Needle and sham needle

\section{Operated side EA group}

The operated side of the lower limbs will be treated with true EA while the healthy side will be treated with sham EA.

\section{Sham EA group}

Sham EA will be used on both the operated and healthy side.

The specific procedures of EA and sham EA are as follows (Fig. 2):

Electroacupuncture (EA) Needles $(0.30 \mathrm{~mm}$ in diameter, $40 \mathrm{~mm}$ in length, Hwato Brand, Suzhou Medical Appliance Factory, China) will be inserted vertically into the acupoints through the adhesive pads to a depth of 25-30 mm (Fig. 3). Acupuncturists will twist to achieve and enhance the sensation of needles inserted into the skin around acupoints, such as numbness, heaviness, aches, etc. (known as De Qi). Subsequent electrical stimulation (SDZ-V electroacupuncture apparatus, Huatuo brand, Suzhou Medical Appliance Factory, China) will be connected to each needle handle (a connecting wire connects a pair of acupoints, GB34 to ST32 and ST36 to SP9) and a continuous wave of $2 \mathrm{~Hz}$ frequency and an intensity of $1 \mathrm{~mA}$ will be maintained.

Sham electroacupuncture (Sham EA) The blunt needle (Fig. 3, $0.30 \mathrm{~mm}$ in diameter, $25 \mathrm{~mm}$ in length, Hwato Brand, Suzhou Medical Appliance Factory, China) will reach the skin through the adhesive pads without penetrating the skin. The needle handle is rotated three times to simulate the real acupuncture procedure. The sham electrical stimulation apparatus looks like the real one, but with the inner metal wire cut off so no current passes through the connecting wire between the two needle handle (GB34 to ST32 and ST36 to SP9); a continuous wave of $2 \mathrm{~Hz}$ frequency and an intensity of $1 \mathrm{~mA}$ is applied [31].

During the treatment, the needle handles are rotated on both sides three times per $5 \mathrm{~min}$ by acupuncturist.

\section{Outcome measures}

\section{Primary outcome measurement}

The main evaluation criterion is pain, which is divided into two parts.

The first part is a visual analogue scale (VAS). The assessment schedule is shown in Table 1. Independent assessors who are not involved in acupuncture treatments will perform the VAS assessments (asking subjects to indicate the intensity of pain at the most painful points while resting and walking). The $0-100 \mathrm{~mm}$ VAS is used to evaluate pain intensity, wherein $0 \mathrm{~mm}$ denotes painless and $100 \mathrm{~mm}$ represents the most intolerable pain [32]. It allows patients to mark their own feelings on the scale as the evaluation score of pain. In addition to evaluating resting pain at $6 \mathrm{~h}$ after the operation, the pain in both the resting and active state is measured immediately after treatment on the first to third days after the operation, and assessed at follow-up on the fifth, seventh, and tenth days after the operation.

The second part is to record any additional doses from the patient-controlled analgesic pump after the operation, and to evaluate it uniformly on the second day after the operation (at $24 \mathrm{~h}$ after the first treatment).

\section{Secondary outcome measurements}

The secondary outcome measurements are mainly related to rehabilitation after the TKA operation. They include the following. 
Table 1 Schedule of trial enrollment, interventions, and assessments

\begin{tabular}{|c|c|c|c|c|c|c|c|c|c|}
\hline Items & Enrollment (pre-operative) & Allocation (baseline) & Trea & ent ( & $-o p$ & & Follc & $w-u p$ & \\
\hline Time point (hour/day) & -1 & -1 & $6 \mathrm{~h}$ & $1 d$ & $2 d$ & $3 d$ & $5 d$ & $7 d$ & $10 d$ \\
\hline Treatment sessions ( $n$ ) & - & - & & 1 & 2 & 3 & & & \\
\hline Informed consent & $\sqrt{ }$ & & & & & & & & \\
\hline Assessment of eligibility & $\sqrt{ }$ & & & & & & & & \\
\hline VAS (resting) & & $\sqrt{ }$ & $\sqrt{ }$ & $\sqrt{ }$ & $\sqrt{ }$ & $\sqrt{ }$ & $\sqrt{ }$ & $\sqrt{ }$ & $\sqrt{ }$ \\
\hline VAS (active) & & $\sqrt{ }$ & & $\sqrt{ }$ & $\sqrt{ }$ & $\sqrt{ }$ & $\sqrt{ }$ & $\sqrt{ }$ & $\sqrt{ }$ \\
\hline Additional dose released by PCA pump & & & & & $\sqrt{ }$ & & & & \\
\hline HSS score & & $\sqrt{ }$ & & & & $\sqrt{ }$ & & & $\sqrt{ }$ \\
\hline HAMA score & & $\sqrt{ }$ & & & & $\sqrt{ }$ & & & $\sqrt{ }$ \\
\hline AROM & & $\sqrt{ }$ & & & & $\sqrt{ }$ & $\sqrt{ }$ & $\sqrt{ }$ & $\sqrt{ }$ \\
\hline PROM & & $\sqrt{ }$ & & & & $\sqrt{ }$ & $\sqrt{ }$ & $\sqrt{ }$ & $\sqrt{ }$ \\
\hline COK & & & & $\sqrt{ }$ & & $\sqrt{ }$ & $\sqrt{ }$ & $\sqrt{ }$ & $\sqrt{ }$ \\
\hline Discomfort and acceptance of EA & & & & $\sqrt{ }$ & $\sqrt{ }$ & $\sqrt{ }$ & & & \\
\hline Assessment of blinding method & & & & & & $\sqrt{ }$ & & & \\
\hline Postoperative complications and adverse events & & & & $\sqrt{ }$ & $\sqrt{ }$ & $\sqrt{ }$ & & & \\
\hline
\end{tabular}

VAS Visual Analogue Score, PCA patient controlled analgesia, HSS hospital for special surgery, HAMA Hamilton Anxiety Scale, COK circumference of knee, AROM active range of motion, $P R O M$ passive range of motion

The knee score of patients will be measured at three time points-the day before operation and on the third and tenth days after the operation-using the Hospital for Special Surgery Knee Score (HSS), commonly used in similar studies. The HSS gives a maximum of 100 points and includes pain (30 points), function (22 points), range of motion (18 points), muscular strength (10 points), deformity (10 points), instability (10 points), and subtractions (three items) [33, 34].

Active and passive range of motion (ROM) of knee joint will be measured by a standard joint protractor measure [35] on the day before the operation and the third, fifth, seventh, and tenth days after the operation.

Based on the ratio of knee circumference on the first day after the operation, the improvement of swelling around the knee joint will be evaluated on the third, fifth, seventh, and tenth days after the operation according to the following formula: (COK on the Xth-COK on the first day after operation) /(COK on first day after operation), where $\mathrm{COK}$ is the circumference of the knee at suprapatellar pole20 [20].

The Hamilton Anxiety Scale (HAMA) consists of 14 items, each of which has five levels [36]. In this study, the severity of anxiety in patients will be assessed on the day before the operation, and the third and tenth days after the operation.

\section{Assessment of the subject blinding success rate for acupuncture}

Patients of the three groups will be asked to guess their assigned group to assess the masking effectiveness of the trial (Table 2), at $5 \mathrm{~min}$ after the end of the third treatment. Subjects will be asked the following questions: (1) How does the needle sensation feel (sensations induced by acupuncture, like sourness, numbness, distending, heaviness, etc.)? Please ring a number between 0 (no needle sensation) and 10 (unbearable needle sensation). (2) Have you ever received acupuncture? (3) Have you ever received electroacupuncture? (4) Do you think you have been treated at acupoints? (5) Are you sure you are receiving acupuncture treatment? [37] (6) At which lower extremity do you think you have been needled?

\section{EA safety and adverse events}

Although acupuncture has been deemed a safe and reliable therapy, its adverse effects and complications associated should raise concerns [12]. Clinically, the incidence of minor adverse events related to acupuncture may be higher, such as pain, local infection, fatigue, local hematoma, subcutaneous hemorrhage, etc. [38, 39]. Some of these might be avoidable [40]. During the treatment period, acupuncturists will strictly abide by the operating rules of electroacupuncture [41]. All acupoints are around the knee joint(s). Acupuncture insertion is in the superficial or subcutaneous regions. Occasionally, there will be a small amount of bleeding or bruising. The bleeding can be arrested by pressing with sterile cotton swabs or cotton balls. The bruising usually disappears within 2-3 days [32].

In this study, during the entire clinical trial, observers who are also blinded to group allocation will record the adverse events (AEs) related to acupuncture treatment in detail and enumerate the incidences. AEs will be 
Table 2 Blind method assessment questionnaire

\begin{tabular}{l}
\hline \multicolumn{1}{c}{ Blind Method Assessment Questionnaire } \\
\hline Name: Dandom number: \\
Please answer the following questions according to your latest acupuncture experience. \\
1.How does the needle sensation feel (sensations induced by acupuncture, like sourness, numbness, \\
distending, heaviness, etc)? \\
Please ring a number between 0 (no needle sensation) and 10 (unbearable needle sensation).
\end{tabular}

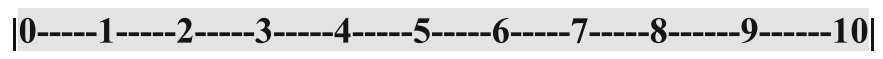

2.Have you ever received acupuncture?

3.Have you ever received electroacupuncture?

4.Do you think you have been treated at acupoints?

5.Are you sure you are receiving acupuncture treatment?

6.At which lower extremity do you think you have been needled?

$\begin{array}{ll}(\text { Yes } & \text { No }) \\ (\text { Yes } & \text { No }) \\ (\text { Yes } & \text { No }) \\ (\text { Yes } & \text { No) }\end{array}$

(Healthy side Operated side Both side)

Note: The questionnaire survey will be completed within 5 min after the end of the third treatment

managed by acupuncturists and relevant clinical experts within $24 \mathrm{~h}$. Any adverse event, whether related to treatment or not, is reported by the participants and practitioners at every visit.

\section{Ethics and dissemination}

The protocol of this clinical trial adheres to the SPIRIT guidelines. Willingness to be randomly assigned and understanding and signing the informed consent is imperative for every participant in the study. All study participants will agree in writing, and researchers will provide information on this trial in detail to each person. The ethical validity of the study has been assessed and approved by the Institutional Review Board of Guanghua Hospital, Shanghai University of Traditional Chinese Medicine (No.2018-K-18). The study is registered in the Chinese Clinical Trials Registry (trial registration number ChiCTR1800020297), where the website will present the result of the study.

\section{Data management}

In this study, YLC and LBX, with at least 20 years of clinical experience, are the initiators and instructors. $\mathrm{HH}$ is the acupuncturist for the study, ${ }^{4} \mathrm{LZ}$ will be the clinical assessor, and ${ }^{3} \mathrm{LZ}$ and XLS will conduct data management and analyze results. Any changes to the protocol will be approved by the ethics committee. Due to the small sample size and the known low risk of acupuncture [38], a formal data monitoring committee will not be necessary. During the trial, independent investigators in the hospital will regularly monitor and audit the data collected to ensure good data quality. Within one week after completing the data collection, all data will be entered into a password-protected computer by two independent trained research assistants. Finally, CRFs and research documents will be filed in the department research cabinet.

\section{Statistical analysis}

The data in this study will be analyzed using the Statistical Package for Social Science software (SPSS version 22.0, IBM Corp, New York). After data collection, statistical analysis will be performed by a special statistician. Data analysis will be based on the intention-to-treat (ITT) principle [42], considering significance levels < 0.05 as statistically significant.

Continuous data will be represented by mean, standard deviation, median, and interquartile ranges and the categorical data by percentages.

According to whether the data are normally distributed, the analysis of baseline will be performed using a $t$-test or a Wilcoxon rank sum test for continuous data and a chi-squared test or Fisher's exact test for categorical data. Comparisons among the three groups will be analyzed by one-way analysis of variance (ANOVA) [43]. When the distribution is not normal, nonparametric tests will be used. Thus, the primary outcome will be assessed using a one-way, repeated-measures ANOVA to test the influence of the time, the group, and the time"group interaction on pain. Comparisons of categorical data among groups will be tested by the chi-square test or the Mann Whitney U-test. 


\section{Sample size}

According to the data from the previous preliminary study, through observing the analgesic effect of opposing EA three times after TKA operation, the average VAS score was 37.0 with a standard deviation $=22.6$, the average value of sham EA was 46.9 , and the standard deviation was 12.5 , with a significance level $\alpha=0.05$ (twosided) and power $(1-\beta)=0.8$. According to the following sample size formula $[44,45], \mu$ is the mean and $\sigma$ is the standard deviation, $\mathrm{K}=\mathrm{n}_{\mathrm{A}} / \mathrm{n}_{\mathrm{B}}$ is the matching ratio. $\mathrm{T}$ is the number of comparisons to be made. Look-up table $\mathrm{Z}$ can be obtained. The sample size was calculated as 96 cases; taking a $15 \%$ dropout rate into account, 38 subjects are required in each of the three groups.

$$
n_{A}=\left(\sigma_{A}^{2}+\sigma_{B}^{2} / \kappa\right)\left(\frac{Z_{1-\alpha / \tau}+Z_{1-\beta}}{\mu_{A}-\mu_{B}}\right)^{2}
$$

\section{Discussion}

In recent years, with the development of fast-track (FT) surgery for joint problems, clinicians pursue the goals of painless, edema-free, stasis-free, and tubeless rehabilitation management after surgery [46]. For post-operative pain management, we should not only adopt the multimode analgesia model [47], but also promote the development of multi-disciplinary [48] approaches, especially by adding the high-quality analgesic methods of traditional medicine and developing the combination of traditional Chinese medicine and modern clinical medicine [49].

This clinical study of opposing needling on analgesia and rehabilitation after TKA is aimed at understanding the feasibility of such a procedure. Under the unified conditions of routine operation mode and routine intraoperative and post-operative analgesic dosage and on the premise of baseline equilibrium of the three groups, three interventions will be given according to each patient's random number: opposing EA, operated side EA, sham EA.

Acupuncture on the affected side is a conventional non-drug therapy for rehabilitation after TKA [20, 50]. Opposing needling means applying acupuncture on the healthy leg (unoperated) of the patient, where acupoints are selected according to corresponding acupoints and meridian of the operated side. Accumulating evidence from clinical and experimental studies revealed that opposing needling exerts protective and facilitative effects on functional rehabilitation, the underlying mechanism of which remains to be further studied [51]. For patients, needling the healthy side after operation is more convenient and more acceptable. In terms of risk, needling the affected side increases the risk of infection after TKA. At the same time, the opposing needling method avoids the disadvantage of inconvenient selections of acupoints distributed at the surgical site.

In addition to the evaluation of the curative effect during hospitalization, good communication and follow-up with patients will be established. Not only will it help patients physically, but also care for patients psychologically. Through the study, we hope to provide clinicians with high-quality evidence of acupuncture for the recovery of pain and function after TKA and provide more ideas for clinicians in perioperative pain management.

\section{Abbreviations \\ TKA: Total knee arthroplasty; EA: Electroacupuncture; KOA: Knee osteoarthritis; CPSP: Chronic postsurgical pain; SAEs: Severe adverse events; PCA: Patient- controlled analgesic; VAS: Visual analogue scale; CRF: Case report form; ASA: American Society of Anesthesiologists; ST: Stomach meridian of foot- yangming; SP: Foot-taiyin spleen meridian; GB: Gall bladder channel of foot- Shaoyang; HSS: Hospital for special surgery; ROM: Range of motion; COK: Circumference of the knee; HAMA: Hamilton Anxiety Scale; AEs: Adverse events; SPSS: Statistical Package for Social Science; ITT: Intention-to-treat; ANOVA: One-way analysis of variance; FT: Fast-track}

\section{Acknowledgements}

The Shanghai University of Traditional Chinese Medicine provided the requisite funding for the project.

Colleagues in the joint surgery department of Guanghua Hospital, Shanghai University of Traditional Chinese Medicine have provided their professional guidance.

\section{Trial status}

Protocol version number V1.0, 14 November 2018.

Recruitment began on 27 February 2019

The approximate date when recruitment will be completed is 31 December 2019.

\section{Authors' contributions}

YLC and LBX conceived the study, designed the study protocol, and sought ethical approval. HH wrote the final manuscript. LZ contributed to the study design and made critical revisions. XLS and LZ participated in critical revision of this manuscript. All authors read and approved the final manuscript.

\section{Funding}

This study is funded by the Shanghai University of Traditional Chinese Medicine grant Y201908. YL C, LZ, and XL S are from Shanghai University of Traditional Chinese Medicine and helped in study design, data collection and analysis, and writing manuscripts. For the details, please refer to 'Authors' contributions' section.

Address: Shanghai University of Traditional Chinese Medicine, 1200 Cailun Rd. Shanghai 201203, China.

\section{Availability of data and materials}

The datasets will be available upon reasonable request after completion of the study.

\section{Ethics approval and consent to participate}

Willingness to be randomly assigned and understanding and signing the informed consent will be necessary for each participant of the study. All study participants will agree in writing, and researchers will provide information of this trial in detail to each participant. The ethical validity of the study has been assessed and approved by the Institutional Review Board of Guanghua Hospital, Shanghai University of Traditional Chinese Medicine (No.2018-K-18).

\section{Consent for publication}

Not applicable.

Competing interests

The authors declare that they have no competing interests. 


\section{Received: 12 October 2019 Accepted: 13 March 2020} Published online: 07 May 2020

\section{References}

1. Jüni $P$, Reichenbach $S$, Dieppe $P$. Osteoarthritis: rational approach to treating the individual. Best Pract Res Clin Rheumatol. 2006;20(4):721-40.

2. Da Silva RR, Santos AA, de Sampaio Carvalho Júnior J, Matos MA. Quality of life after total knee arthroplasty: systematic review. Rev Bras Ortop. 2014; 49(5):520-7.

3. Fernandes DA, Poeta LS, Martins CAQ, Lima F, Rosa Neto F. Balance and quality of life after total knee arthroplasty. Rev Bras Ortop. 2018;53(6):74753.

4. Clement ND, Macdonald D, Howie CR, Biant LC. The outcome of primary total hip and knee arthroplasty in patients aged 80 years or more. J Bone Joint Surg Br. 2011;93(9):1265-70.

5. Saku SA, Madanat R, Mäkinen TJ. Reasons and risk factors for ninety day readmission following primary total knee arthroplasty in a high-volume centre. Int Orthop. 2018;42(1):95-9.

6. Xiong J, Li H, Li X, Wang L, Zhao P, Meng D, Wei ZX, Tian T. Electroacupuncture for postoperative pain management after total knee arthroplasty: Protocol for a systematic review and meta-analysis. Medicine (Baltimore). 2018;97(9):e0014.

7. Hernandez NM, Parry JA, Taunton MJ. Patients at risk: large opioid prescriptions after total knee arthroplasty. J Arthroplast. 2017;32(8):2395-8.

8. Huang PS, Copp SN. Oral opioids are overprescribed in the opiate-naive patient undergoing total joint arthroplasty. J Am Acad Orthop Surg. 2019. https://doi.org/10.5435/JAAOS-D-18-00404.

9. Kim KY, Anoushiravani AA, Chen KK, Roof M, Long WJ, Schwarzkopf R. Preoperative chronic opioid users in total knee arthroplasty-which patients persistently abuse opiates following surgery? J Arthroplasty. 2018;33(1):10712.

10. De Luca ML, Ciccarello M, Martorana M, Infantino D, Letizia Mauro G, Bonarelli S, Benedetti MG. Pain monitoring and management in a rehabilitation setting after total joint replacement. Medicine (Baltimore). 2018;97(40):e12484.

11. Li JW, Ma YS, Xiao LK. Postoperative pain management in total knee arthroplasty. Orthop Surg. 2019:11(5):755-61.

12. Wu JY, Hu YM, Zhu Y, Yin P, Litscher G, Xu SF. Systematic review of adverse effects: a further step towards modernization of acupuncture in China. Evid Based Complement Alternat Med. 2015;2015:1-19.

13. Chiarioni G, Pesce M, Fantin A, Sarnelli G. Complementary and alternative treatment in functional dyspepsia. United European Gastroenterol J. 2018; 6(1):5-12.

14. Chan YT, Zhang HW, Guo YQ, Chan TNH, Kwan YK, Lee CK, Ngan K, Lin ZX. Effectiveness and safety of acupuncture for elderly overactive bladder population in Hong Kong: study protocol for a randomized controlled trial. Trials. 2018;19(1):376.

15. Liu ZS, Liu Y, Xu HF, He LY, Chen YL, Fu LX, Li N, Lu YH, Su TS, Sun JH, Wang J, et al. Effect of electroacupuncture on urinary leakage among women with stress urinary incontinence: a randomized clinical trial. JAMA. 2017;60(24):2493.

16. Tedesco D, Gori D, Desai KR, Asch S, Carroll IR, Curtin C, McDonald KM, Fantini MP. Drug-free interventions to reduce pain or opioid consumption after total knee arthroplasty. JAMA Surg. 2017;152(10):e172872.

17. Jung J-Y, Cho J-H, Chung $\mathrm{S}-\mathrm{H}$. Acupuncture for postoperative pain following total knee arthroplasty: a systematic review protocol. BMJ Open. 2015;5(11): e009573.

18. Cho Y-H, Kim C-K, Heo K-H, Lee MS, Ha T-H, Son DW, Choi BK, Song G-S, Shin B-C. Acupuncture for acute postoperative pain after back surgery: a systematic review and meta-analysis of randomized controlled trials. Pain Pract. 2015;15(3):279-91.

19. Vickers AJ, Cronin AM, Maschino AC, Lewith G, MacPherson H, Foster NE, Sherman KJ, Witt CM, Linde K. Acupuncture for chronic pain: individual patient data meta-analysis. Arch Intern Med. 2012;55(4):24-5.

20. Mikashima Y, Takagi T, Tomatsu T, Horikoshi M, Ikari K, Momohara S. Efficacy of acupuncture during post-acute phase of rehabilitation after total knee arthroplasty. J Tradit Chin Med. 2012;32(4):545-8.

21. Zhong S, Huang H, Xie J, Zhao L, Song XL, Chen YL, Xiao LB. Application of electroacupuncture for postoperative pain management after total knee arthroplasty: a study protocol for a single-blinded, randomised placebocontrolled trial. BMJ Open. 2019;9(4):e026084.
22. Yang Z-X. Meridians and acupoints with opposite locations. Zhongguo Zhen Jiu. 2011;31(12):1139-41.

23. Ye MZ. Contralateral needling method and its applications in treating upper-limb pain. J Acupunct Tuina Sci. 2012;10(1):58-61.

24. Fan GQ, Wu X, Xue ZY, Mao SZ. Application of healthy-side needling to treatment of apoplectic hemiplegia. J Tradit Chin Med. 2002;22(2):143-7.

25. Tao H-B. Treatment of 53 cases of acute lumbar muscle strain by contralateral needling. J Acupunct Tuina Sci. 2004;2(5):41-2.

26. Gao HM, Li XG, Gao X, Ma BX. Contralateral needling at unblocked collaterals for hemiplegia following acute ischemic stroke. Neural Regen Res. 2013;8(31):2914-22.

27. Yi M, Zhang $H$, Lao L, et al. Anterior cingulate cortex is crucial for contrabut not ipsi-lateral electro-acupuncture in the formalin-induced inflammatory pain model of rats. Mol Pain. 2011;7(1):61.

28. Zhang S, Wang X, Yan C-Q, et al. Different mechanisms of contralateral- or ipsilateral-acupuncture to modulate the brain activity in patients with unilateral chronic shoulder pain: a pilot fMRI study. J Pain Res. 2018;11:50514

29. Saklad M. Grading of patients for surgical procedures. Anesthesiology. 1941; 2:281-4.

30. He GH, Ruan JW, Zeng YS, Zhou X, Ding Y, Zhou GH. Improvement in acupoint selection for acupuncture of nerves surrounding the injury site: electro-acupuncture with Governor vessel with local meridian acupoints. Neural Regen Res. 2015;10(1):128-35.

31. Qin Z-S, Zang Z-W, Wu J-N, Zhou J, Liu Z-S. Efficacy of acupuncture for chronic prostatitis /chronic pelvic pain syndromes: study protocol for a randomized, sham acupuncture-controlled trial. BMC Complement Altern Med. 2016;16(1):440.

32. Au KY, Chen H, Lam WC, Chong CO, Lau A, Vardhanabhuti V, et al. Sinew acupuncture for knee osteoarthritis: study protocol for a randomized shamcontrolled trial. BMC Complement Altern Med. 2018;18:133.

33. Narin S, Unver B, Bakırhan S, Bozan O, Karatosun V. Cross-cultural adaptation, reliability and validity of the Turkish version of the Hospital for Special Surgery (HSS) Knee Score. Acta Orthop Traumatol Turc. 2014;48(3):241-8.

34. Tekin B, Unver B, Karatosun V. Expectations in patients with total knee arthroplasty. Acta Orthop Traumatol Turc. 2012;46(3):174-80.

35. Dos Santos RA, Derhon V, Brandalize M, Brandalize D, Rossi LP. Evaluation of knee range of motion: correlation between measurements using a universal goniometer and a smartphone goniometric application. J Bodyw Mov Ther. 2017;21(3):699-703.

36. Euan T. Hamilton Rating Scale for Anxiety (HAM-A). Occup Med (Lond). 2015;65(7):601.

37. Liu ZS, Xu HF, Chen YL, He LY, Liu J, Yan SY, et al. The efficacy and safety of electroacupuncture for women with pure stress urinary incontinence: study protocol for a multicenter randomized controlled trial. Trials. 2013;14(1):1-9.

38. Macpherson $\mathrm{H}$, Thomas $\mathrm{K}$, Walters $\mathrm{S}$, Fitter M. A prospective survey of adverse events and treatment reactions following 34,000 consultations with professional acupuncturists. Acupunct Med. 2002;19(2):93-102.

39. Yamashita H, Tsukayama H, Hori N, Kimura T, Tanno Y. Incidence of adverse reactions associated with acupuncture. J Altern Complement Med. 2000; 6(4):345-50.

40. White A. A cumulative review of the range and incidence of significant adverse events associated with acupuncture. Acupunct Med. 2004;22(3): 122-33.

41. Wang P, Yang H-Y, Hu Y-E. Compilation experience of national standard Standardized Manipulation of Acupuncture and Moxibustion, Part 11, Electroacupuncture. Zhongguo Zhen Jiu. 2010;30(5):413-6.

42. Detry M-A, Lewis R-J. The Intention-to-Treat Principle. JAMA. 2014;312(1):856.

43. Eunsik P, Meehye C, Chang-Seok K. Correct use of repeated measures analysis of variance. Korean J Lab Med. 2009;29(1):1-9.

44. Glueck DH. Sample size calculations in clinical research, 2nd edition by Chow, S.-C., Shao, J., and Wang, H. Biometrics. 2008;64(4):1307-8.

45. Rosner B. Fundamentals of biostatistics. 7th ed. Belmont: Duxbury Press; 2010. p. 302-3.

46. Ansari D, Gianotti L, Schröder J, Andersson R. Fast-track surgery: procedurespecific aspects and future direction. Langenbeck's Arch Surg. 2013;398(1): 29-37.

47. Lewis C, Gunta K, Mitchell K, Bobay K. Effectiveness of multimodal pain management protocol in total knee arthroplasty patients. Orthop Nurs. 2012;31(3):153-9. 
48. Feng JE, Novikov D, Anoushiravani AA, Schwarzkopf R. Total knee arthroplasty: improving outcomes with a multidisciplinary approach. J Multidiscip Healthc. 2018;11:63-73.

49. Zhang JH, Wu MS, Wang YF, Jia YM, Li E. Medicine in future and advantages of integrated Chinese and Western medicine. Chin J Integr Med. 2019;25(2): 87-90.

50. Tzeng CY, Chang SL, Wu CC, Chang CL, Chen WG, Tong KM, Huang KC, Hsieh CL. Single-blinded, randomised preliminary study evaluating the effects of $2 \mathrm{~Hz}$ electroacupuncture for postoperative pain in patients with total knee arthroplasty. Acupunct Med. 2015;33(4):284-8.

51. Jiang YJ, Yang SL, Tao J, Lin ZC, Ye XQ, You YM, Peng J, Hong ZF, Chen LD. Opposing needling promotes behavior recovery and exerts neuroprotection via the CAMP/PKA/CREB signal transduction pathway in transient MCAO rats. Mol Med Rep. 2016;13(3):2060-70.

\section{Publisher's Note}

Springer Nature remains neutral with regard to jurisdictional claims in published maps and institutional affiliations.

Ready to submit your research? Choose BMC and benefit from:

- fast, convenient online submission

- thorough peer review by experienced researchers in your field

- rapid publication on acceptance

- support for research data, including large and complex data types

- gold Open Access which fosters wider collaboration and increased citations

- maximum visibility for your research: over $100 \mathrm{M}$ website views per year

At $\mathrm{BMC}$, research is always in progress.

Learn more biomedcentral.com/submissions 